\title{
Cross-domain Recommendations for Personalized Semantic Services
}

\author{
Hla Hla Moe \\ University of Technology \\ Yatanarpon Cyber City, \\ Pwin Oo Lwin, Myanmar
}

\author{
Win Thanda Aung \\ University of Computer Studies \\ Bahan Campus, \\ Yangon, Myanmar
}

\begin{abstract}
An increasing amount of work has been published in various areas related to the Recommender System. Among them, cross-domain recommendation is an emerging research topic and in this field, it is important to investigate how to manage personalization and how to consider customer's contextual features to keep more user satisfaction and accuracy. This paper tends to provide cross-domain recommendations for personalized semantic services using Taxonomic CCBR, directed acyclic graph by FordFulkerson algorithm and TOPSIS method. Taxonomic CCBR helps the system get the accurate problem by engaging a user in a series of questions and answers from the user's partial definition of the problem. Semantic concepts between different domains are considered by using weighted directed acyclic graph to find meaningful solutions. Then TOPSIS method is used to get the results more precisely considering contextual features such as season, place, etc. which have not been addressed in the current cross-domain recommender systems.
\end{abstract}

Keywords: recommender systems; cross-domain recommendations; personalization; semantic concepts; customer's contextual features

\section{INTRODUCTION}

Through the Internet, different products, services and customers can now easily interact with each other because the advance of Internet and Web technologies has continuously boosted the prosperity of e-commerce [4]-[7]. On the other hand, the more continuous development of electronic commerce, the more difficult it is for customers to single out products or services and find the most suitable ones with them. To make a suitable decision, customers still spend much time in visiting a flood of online retailers, and gather valuable information by themselves. This process is very timeconsuming and sometimes the contents of Web documents that customers browse have nothing to do with those that they need indeed.

To overcome the above problem, recommender system is a main solution. Recommender systems typically provide the user with a list of recommended items they might prefer, or supply guesses of how much the user might prefer each item [2]-[4]. Such systems are now popular both commercially and in the research community and even overwhelm human processing capabilities in a wide array of information seeking for decision making. So many Web sites such as Amazon, Netflix, Last.fm and many online retailers have proved that recommendation models are successful [2]-[7]. However, ample room and need for further improvements remain in the effective human decision support in a wide variety of applications.

The vast majority of these systems offer their recommendations only for items belonging to a single domain. In fact, joint recommendations in multiple domains are sometime needed for a customer. For instance, a system suggests not only a particular movie but also music CDs and books that are somehow related to that movie [2]-[7]-[5]-[12]. Such types are cross-domain recommendations. By definition, cross-domain recommendation is providing recommendations of items in one (source) domain using the preferences expressed on items in a second (target) domain [7]-[5]. Another task for cross-domain recommendation is making joint recommendations for items belonging to different domains [13].

According to [2], cross-domain recommendation models are classified into adaptive models - which exploit information directly from a source domain to make recommendations in a target domain - and collective models - which are built with data from several domains and potentially can make joint recommendations for such domains. Generally, almost all cross-domain recommendations are less accurate than single-domain recommendations but they can give more diverse recommendations leading to a higher user satisfaction and engagement addressing cold-start and sparsity problems [5][7]-[11]-[13]-[15].

In this paper, a framework for cross-domain recommender system is proposed. Previous cross-domain recommender systems are constructed based on music, music artists and related things [5]-[7]-[11]-[17]. The framework is used to apply the useful application area where skin care problem is solved and suitable cosmetics are recommended. The framework tends to solve customer's personalized problem and give more accurate recommendations in a particular way. To handle personalization systematically, Taxonomic CCBR is used. It allows for a partial definition of the problem by the user, identifies more clearly user's problem and gives accurate solution by conversation. Hence, it improves personalization. To extract the semantic concepts between domains, the Ford and Fulkerson algorithm is applied by directed acyclic graph. Consideration of customer's contextual features such as season, place and so on is an important role for managing personalization. And hence, to get personalized recommendations precisely and accurately. TOPSIS method is used to calculate the final results considering contextual features.

The rest of the paper is organized as follows. Section 2 describes our motivation. In section 3 , related works on crossdomain recommender systems are briefly described. In section 4 , system overview of the proposed system is described. Section 5 also describes detailed approach for cross-domain 
recommendations for personalized semantic services and section 6 describes the conclusion.

\section{MOTIVATION}

In the most current recommender systems, recommendations are only from a single domain. Therefore, today, crossdomain recommender systems become an interesting topic. This type of recommendations has barely been investigated because it is difficult to obtain public datasets with user preferences crossing different domains. The previous related works for cross-domain recommendations still have some weaknesses. Some are lack of personalization. Some are lack of semantic concepts and less accuracy. Our framework tends to solve these weaknesses. And then the objectives of our framework are to understand about the advantages of crossdomain recommender systems adding semantic concepts, to get more accurate, precise and personalized recommendations and to know the interesting results of cross-domain recommendations applying TOPSIS method with customer's contextual features.

\section{RELATED WORK}

The application of cross-domain recommendation approaches becomes of special interest in many e-commerce and retailer websites because it can increase company profits and strengthen customers' loyalty. Therefore, cross-domain recommendation is an interesting research area and applies in many application areas, and even mobile environments. In the previous works, cross-domain recommender systems have been proposed in various kinds of ways.

Gustavo González et al. described an approach for crossdomain recommender systems using Smart User Model (SUM). It was a multi-agent based system. It used incremental aggregation of information, which favored non-intrusive behavior of the user model in order to determine objective, subjective and emotional user features [12].

A generic framework to mediate the integration of data collected by several recommender systems was presented by Shlomo Berkovsky et al. They discussed four major types of mediation: cross-user, cross-item, cross-context, and cross representation. Some evaluations had shown that in certain conditions, user modeling data mediation improved the quality of recommendations, especially in the cold start of a recommender system [10].

References [17] and [5] showed that Marius Kaminskas and Francesco Ricci proposed an approach which considered contextual conditions such as the user mood or location. It retrieved music that suited the user's interested place using emotional tags attached by users' population to both music and POIs. It applied a set of similarity metrics for tagged resources to establish a match between music track and POIs.

Francesco Ricci et al. designed and developed a generic framework built upon semantic networks, which integrated and exploited knowledge on several domains to provide crossdomain adapted item recommendations. They proposed an approach that automatically extracted information about two domains available in Linked Data repositories, linked items in the two domains by means of a weighted directed acyclic graph, and performed weight spreading mechanisms on such graph to identify matching items in a target domain (music artists) from items of a source domain (places of interest) [7].

Fabian Abel et al. studied distributed form-based and tagbased user profiles, based on a large dataset aggregated from the Social Web. The performance of several cross-system user modeling strategies in the context of recommender systems is developed and evaluated to solve the cold-start problem and improve recommendation quality [13].
Jie Tang et al. proposed Cross-domain Topic Learning (CTL) approach to address three challenges: sparse connections - cross-domain collaborations were rare, complementary expertise - cross-domain collaborators often have different expertise and interest and topic skewness cross-domain collaboration topics are focused on a subset of topics. For handling sparse connections, CTL consolidates the existing cross-domain collaborations through topic layers instead of at author layers, which alleviated the sparseness issue. For handling complementary expertise, CTL models topic distributions from source and target domains separately, as well as the correlation across domains. For handling topic skewness, CTL models only relevant topics to the crossdomain collaboration [15].

\section{SYSTEM OVERVIEW OF CROSS- DOMAIN RECOMMENDATIONS FOR PERSONALIZED SEMANTIC SERVICES}

To provide cross-domain recommendations for personalized semantic services, recommendation algorithm works as follows:

1. User gives the initial dialog from the system interface.

2. Taxonomic CCBR system defines the definite problem.

3. According to the problem, the concepts from the source and target domains are linked by means of weighted directed acyclic graph for semantic filtering.

4. From the results, the vectors of product and customer's contextual features are constructed and the utility value of the each product is calculated using TOPSIS method.

5. Recommendations are given to the user.

System overview of cross-domain recommendations for personalized semantic services can be seen as the following figure.

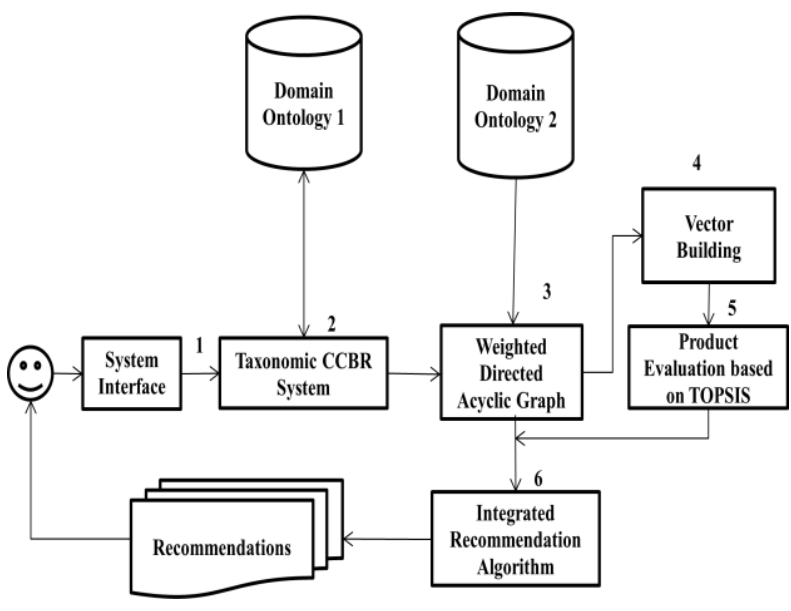

Figure. 1 System overview of cross-domain recommendations for personalized semantic services

\subsection{Algorithm of cross-domain recommendations for personalized semantic services}

Algorithm: Product Recommendation ( )

Input:

user query, customer's contextual features and weights of commodities

Output: 
Init (Q); Load (Q); // initiate and load user query

Begin

DefineProblem $(\mathrm{Q}, \mathrm{c})$; /*define definite problem with Taxonomic CCBR system*/

for each $P_{i} d o$

\{

FindSemanticRelation $\left(\mathrm{c}, \mathrm{P}_{\mathrm{i}}\right) ; / *$ find semantic relation between from problem domain and each product from product domain*/

ComputeWeight $\left(\mathrm{P}_{\mathrm{i}}\right) ; / *$ compute weight of each product using Ford-Fulkerson algorithm*/

InsertTo(ProductList); /* insert $\mathrm{P}_{\mathrm{i}}$ into Product list in descending order*/

\}

while (ProductList != Null)

\{

GetProductUtility $\left(\mathrm{P}_{\mathrm{i}}\right)$; /* compute the utility value of each product from the product list with customer's contextual features*/

InsertTo(ProductList); /* insert $\mathrm{P}_{\mathrm{i}}$ into Product list in descending order*/

\}

Output (ProductList, top-k); $/^{*}$ output top-k products as the recommended product to target customer*/

End

\section{CROSS-DOMAIN \\ RECOMMENDATIONS FOR}

PERSONALIZED SEMANTIC SERVICES

To obtain cross-domain recommendations for personalized semantic services, the framework is considered as three stages and worked as follows.

\subsection{Acquiring Definite Problem from User Query}

In this stage, Taxonomic conversational case-based reasoning (Taxonomic CCBR) is applied. the user is not expected to know exactly which type of problem she has but she is required to answer a set of questions such that the system identifies more clearly what her problem is [3]-[6]. Given information related to the domain, the retrieval process is initiated whereby all questions in taxonomy relevant to that particular domain are presented to the user. Given the set of questions to choose from, the user can then decide to answer some of these questions. Depending on the answers provided, the system will try to find cases in which questions were answered in a similar manner. A similarity measure is used to rank cases. The questions which are present in the retrieved cases but which are still unanswered, yet are related to the problem, are then presented in a rank order to the user. The process continues until the system gets a case which includes a definite problem, personalizing the solution to her needs.

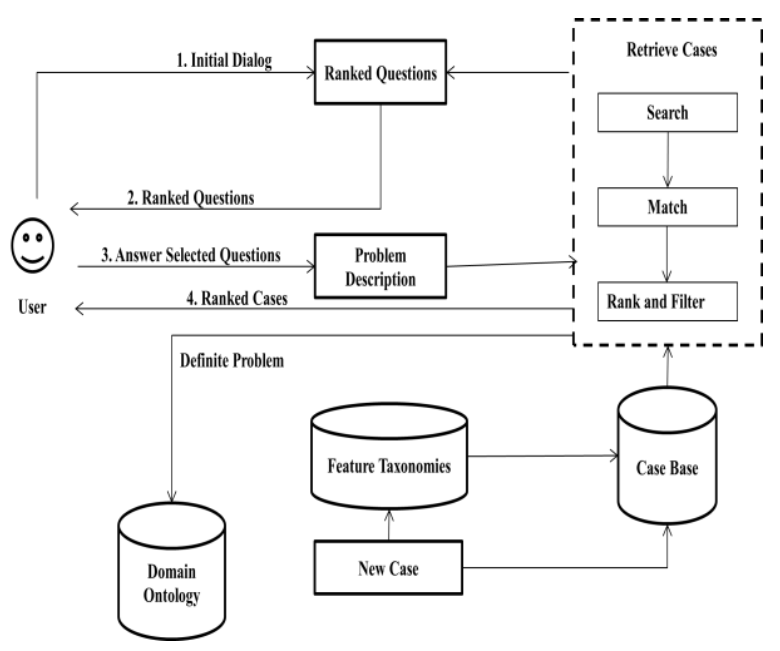

Figure. 2 User interactions with a Taxonomic CCBR system

For case retrieval, Taxonomic theory is divided into two steps taking into account that each question-answer (QA) pair is a set of triples or rather an acyclic directed graph:

(i) similarity between QA pairs

$$
\operatorname{sim}\left(C_{q 1}, C_{q 2}\right)=\left\{\begin{array}{cr}
1 & i f C_{q 1} \subseteq C_{q 2} \\
(n+1-m) /(n+1+m) & i f C_{q 2} \subseteq C_{q 1} \\
0 & \text { otherwise }
\end{array}\right.
$$

where,

$C_{q 1}$ and $C_{q 2}$ are concepts

$n=$ number of edges between $C_{q l}$ and the root

$m=$ number of edges between $C_{q 1}$ and $C_{q 2}$

(ii)

an aggregate similarity between the user query $Q$ and a case problem description $P$ to retrieve the most suitable cases

$$
\operatorname{sim}(Q, P)=\frac{\sum_{i \in Q, j \in P} \operatorname{sim}\left(C_{q i}, C_{q j}\right)}{T}
$$

where, $T$ represents the number of taxonomies.

\subsection{Finding Cross-domain Recommendations}

In the second stage, the system finds the semantic relation between source and target domains according to the definite problem from the previous stage in with weighted directed acyclic graph.

Firstly, semantic relationships between domains are considered based on ontology knowledge representation which can be defined as a network (DAG) of semantic entities (concepts) of different domains interlinked by semantic relations (properties). Entities can be categorized as classes (e.g. cosmetic) and instances (e.g. Revlon Toner). Semantic relations are taken into account using the generic ontology rules such as generalization/specification ("is a" relation), aggregation/decomposition ("has a" relation), similarity (e.g. acne, pimple) and relatedness (e.g. "hydroxyl acid" makes acne "clear"). The relationships can link classes (e.g. "cosmetic" consists of "ingredients"), instances (e.g. "tea tree oil" contains "vitamin A") and both types (e.g. "Revlon Toner" is a "cosmetic"). 
In calculation, the weight of relations between instances is identified. [16]-[18] According to Kirchkoff's Law, "everything that leaves the source must eventually get to the sink", how much flowing into the weight of each target node with Ford-Fulkerson algorithm. It is simple and gives accurate weight of each target node. The weight of target node is calculated by

$$
W\left(v_{i}\right)=\sum_{k=1}^{n} f_{k, i}, i>1
$$

where, $n$ is the number of vertices and $f$ is the weight of the flow.

The more the weight is, the better the performance of semantic relation between different domains.

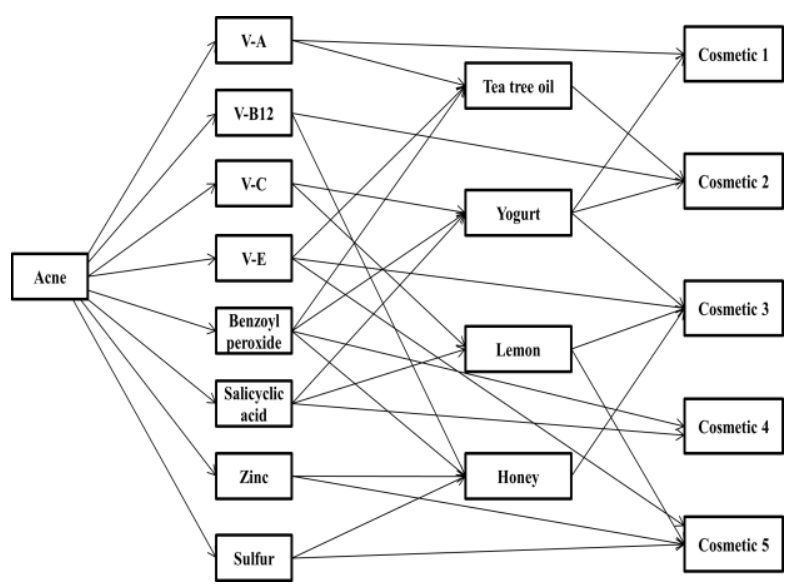

Figure.3 Example of finding semantic relationship between source and target domains by DAG

\subsection{Considering Contextual Features}

Finally, the system calculates the utility value of each candidate product for customer utilizing TOPSIS method, considering customer's contextual features. TOPSIS method is a multi-attribute decision making approach and stands for technique for ordering preference by similarity to ideal solution [1]-[4]-[8]. It is based on the principle that the solution should have the shortest distance to the best solution and the farthest distance to the worst one. As the mathematical model, $P=\left\{p_{1}, p_{2}, \ldots, p_{m}\right\}$ is defined as the vector of the product information and $F=\left\{f_{1}, f_{2}, \ldots, f_{3}\right\}$ is defined as the vector of the customer's contextual features. To represent the relevance performance of the product $p_{i}$ in the qualitative feature $i$, decision matrix can be constructed as the following:

$$
D=\left[\begin{array}{cccc}
d_{11} & d_{12} & \ldots & d_{1 n} \\
d_{21} & d_{22} & \ldots & d_{2 n} \\
\ldots & \ldots & \ldots & \ldots \\
d_{m 1} & d_{m 2} & \ldots & d_{m n}
\end{array}\right]
$$

The decision matrix should be normalized following the formula:

$$
b_{i j}=d_{i j} / \sqrt{\sum_{j=1}^{n} d_{i j}^{2}}, i=1,2, \ldots, m, j=1,2, \ldots, n
$$

The normalized value $b_{i j}$ is limited in $[0,1]$. The utility value of the product $p_{i}$ can then be calculated using the formula:

$$
R_{i}=t_{i}^{-} /\left(t_{i}^{-}+t_{i}^{+}\right), i=1,2, . ., m
$$

where,

$$
\begin{aligned}
& t_{i}^{+}=\sqrt{\sum_{j=1}^{n}\left(c_{i j}-c_{j}^{+}\right)^{2}}, i=1,2, \ldots, m \\
& t_{i}^{-}=\sqrt{\sum_{j=1}^{n}\left(c_{i j}-c_{j}^{-}\right)^{2}}, i=1,2, \ldots, m
\end{aligned}
$$

In the above equations, $\mathrm{n}$ is the number of customer's contextual features, $c_{i j}$ is the weighted normalized decision matrix which is calculated by

$$
c_{i j}=w_{j} b_{i j}, i=1,2, \ldots, m, j=1,2, \ldots, n
$$

where, $w_{j}$ means the customer's relative need in this feature and $c_{j}^{+}, c_{j}^{-}$are the positive and negative ideal solutions:

$$
\begin{aligned}
& C^{+}=\left\{c_{1}^{+}, c_{2}^{+}, \ldots, c_{n}^{+}\right\}=\left\{\left(\max _{i} c_{i j} \mid j \in I\right),\left(\min _{i} c_{i j} \mid j \in J\right)\right\} \\
& C^{-}=\left\{c_{1}^{-}, c_{2}^{-}, \ldots, c_{n}^{-}\right\}=\left\{\left(\min _{i} c_{i j} \mid j \in I\right),\left(\max _{i} c_{i j} \mid j \in J\right)\right\}
\end{aligned}
$$

The more increase the relative closeness $R_{i}$, the more important the utility value of the product $p_{i}$.

Finally, by performing the three stages systematically, the algorithm recommends a ranked list with the highest weighted target instances and the customer can obtain the most suitable products and services that matches her personal problem with full of satisfaction.

\section{CONCLUSION}

The paper presents an approach of cross-domain recommendations for personalized semantic service based on Taxonomic CCBR, Ford-Fulkerson algorithm and TOPSIS method. The system tends to build the framework for recommending cosmetics (target domain) related to customer's skin care problems (source domain) because skin care is the most interesting area for people today. The system is user-friendly and more accurate than the other related works. It gives more personalized semantic recommendations and makes more profits for commercial sites. Therefore, the system becomes an interesting and successful recommender system taking the advantages of ground-truth theory and application area.

\section{ACKNOWLEDGMENTS}

My Sincere thanks to my supervisor Dr. Win Thanda Aung, Lecturer, University of Computer Studies (Bahan Campus), Myanmar for providing me an opportunity to do my research work. I express my thanks to my Institution namely University of Technology (Yatanarpon Cyber City) for providing me with a good environment and facilities like internet books, computers and all that as my source to complete this research. My heart-felt thanks to my family, friends and colleagues who have helped me.

\section{REFERENCES}

[1] Wei J., "TOPSIS Method for Multiple Attribute Decision Making with Incomplete Weight Information in Linguistic," Journal of Convergence Information Technology, Volume 5, Number 10, December 2010, doi: $10.4156 /$ jcit.vol5. issue 10.23

[2] Fernández-Tobías I., Cantador I., Kaminskas M. and Ricci F., "Cross-domain recommendr sysems: A servey of the State of the Art", Escuela Politécnica Superior, Universidad Autónoma de Madrid, 28049 Madrid, Spain 
and Faculty of Computer Science,Free University of Bozen-Bolzano, 39100 Bolzano, Italy, 2012

[3] Abela C. and Montebello M., "PreDiCtS: A Personalised Service Discovery and Composition Framework", Department of Computer Science and AI, University of Malta, 2006

[4] Zeng Z., "A Personalized Product Recommender System based on Semantic Similarity and TOPSIS Method", Journal of Convergence Information Technology, Volume 6, Number 7, July 2011, doi: 10.4156/jcit.vol6.issue7.39

[5] Kaminskas M. and Ricci F., “ Location Adapted Music Recommendation Using Tags", Free University of Bolzano, Piazza Domenicani 3, 39100 Bolzano, Italy, 2011

[6] Moy Gupta K., "Taxonomic Conversational Case-Based Reasoning", Proc. ICCBR 2001, LNAI 2080. Pp.219133,2001

[7] Fernández-Tobías I., Cantador I., Kaminskas M. and Ricci F., "A Generic Semantic-based Framework for Cross-domain Recommendation", Escuela Politécnica Superior, Universidad Autónoma de Madrid, 28049 Madrid, Spain and Faculty of Computer Science,Free University of Bozen-Bolzano, 39100 Bolzano, Italy, 2011

[8] Jadidi O., Firouzi F. andBagliery E., "TOPSIS Method for Supplier Selection Problem", World Academy of Science, Engineering and Technology 71, 2010

[9] Asanov D., "Algorithms and Methods in Recommender Systems", Berlin Institute of Technology, Berlin, Germany, 2011
[10] Berkovsky S., Kuflik T. and Ricci F., "Mediation of User Models for Enhanced Personalization in Recommender Systems", University of Haifa, Haifa, Israel, Free University of Bozen-Bolzano, Italy, 2008

[11] Azak M., "Crossing: A Framework To Develop Knowledge-based Recommenders In Cross Domains", MSc thesis, Middle East Technical University, 2010

[12] González G., López B. and Lluís de la Rosa J., "A Multiagent Smart User Model for Cross-domain Recommender Systems,", Poc. Of IUI 2005 Beyond Personalization Workshop, pp.93-94, 2005

[13] Abel F. , Herder E., Houben G., Henze N. and Krause D., "Cross-system User Modeling and Personalization on the Social Web", Web Information Systems, TU Delft, The Netherlands and IVS Semantic Web Group \& L3S Research Center, Leibniz University Hannover, Germany, 2011

[14] Berkovsky S., Kuflik T. and Ricci F., "Mediation of User Models for Enhanced Personalization in Recommender Systems", University of Haifa, Haifa, Israel and Free University of Bozen-Bolzano, Italy, 2008

[15] Tang J., Wu S., Sun J., and Su H., "Cross-domain Collaboration Recommendation", Department of Computer Science and Technology, Tsinghua University, IBM TJ Watson Research Center, USA, August, 2012

[16] Vatter V., "Graphs, Flows and the Ford-Fulkerson Algorithm", August 12, 2004

[17] Kaminskas M. and Ricci F., "Location Adapted Music Recommendation Using Tags", Free University of Bolzano, Piazza Domenicani 3, 39100 Bolzano, Italy, 2009

[18] http://en.wikipedia.org/wiki/Ford-Fulkerson_algorithm 